\title{
CALIBRATION OF SPACEBORNE POLARIMETRIC SAR DATA USING A GENETIC ALOGRITHM
}

\author{
Toshifumi Moriyama
}

\author{
Department of Electrical and Electronic Engineering, Faculty of Engineering, Nagasaki University \\ E-mail: t-moriya@nagasaki-u.ac.jp
}

\begin{abstract}
Calibration method for spaceborne polarimetric SAR data using a genetic algorithm is discussed in this paper. Recently some satellites which have polarimetric synthetic aperture radar were launched, and the polarimetric data analysis techniques are being developed for terrain classification, forest biomass and soil moisture estimations, etc. Thus, polarimetric calibration becomes an important issue for accurate polarimetric analysis. However, typical polarimetric calibration methods have some restrictions. For example, Freeman method requires the polarimetric data which satisfy reflection symmetry and does not estimate cross-talks. Thus, it is desired that a polarimetric calibration method is needed to estimate all polarimetric calibration parameters by using polarimetric data without considering reflection symmetry. In this report, a polarimetric calibration technique based on a genetic algorithm (GA) is proposed. This proposed method can estimate cross-talks, channel imbalances and Faraday rotation angle using one trihedral corner reflector and the measured polarimetric SAR data with non-reflection symmetry.
\end{abstract}

Index Terms - Polarimetry, synthetic aperture radar (SAR), calibration, genetic algorithm (GA), Faraday rotation

\section{INTRODUCTION}

Recently, some spaceborne polarimetric synthetic aperture radars, such as TerraSAR X (X-band), RADARSAR-2 (C-band) and PALSAR (L-band), are available to measure a scattering matrix of terrain, and polarimetric data analysis techniques are being developed for terrain classification, forest biomass and soil moisture estimations, etc. Thus, polarimetric calibration is necessary to obtain the accurate results from the polarimetric SAR data [1]-[3]. In the case of spaceborne SAR, Faraday rotation affects a measurement of the polarimetric SAR data and its polarimetric calibration becomes complicated procedure as compared with a polarimetric calibration of an airborne polarimetric SAR. The calibration parameters are channel imbalance, cross-talk and Faraday rotation angle.
Freeman proposed the polarimetric calibration method to estimate channel imbalance and Faraday rotation angle under the condition that cross-talk is negligible and the polarimetric data has the reflection symmetry property [4]. In this paper, I propose a polarimetric calibration method to estimate all calibration parameters using a genetic algorithm [5] and polarimetric SAR data does not need to satisfy the reflection symmetry. In section 2, polarimetric calibration model affected by Faraday rotation is addressed. Next, polarimetric calibration method using the genetic algorithm is explained. Finally, we examine that the proposed method is applied to the actual spaceborne polarimetric SAR data.

\section{POLARIMETRIC CALIBRATION MODEL AFFECTED BY FARADAY ROTATION}

For a spaceborne SAR system using linear horizontal $(H)$ and vertical $(V)$ polarizations, the polarimetric calibration model can be written as

$$
\begin{aligned}
\mathbf{M}= & \mathbf{R F S F T}+\mathbf{n} \\
\left(\begin{array}{ll}
M_{H H} & M_{H V} \\
M_{V H} & M_{V V}
\end{array}\right) & =\left(\begin{array}{cc}
1 & \delta_{1} \\
\delta_{2} & f_{1}
\end{array}\right)\left(\begin{array}{cc}
\cos \Omega & \sin \Omega \\
-\sin \Omega & \cos \Omega
\end{array}\right)\left(\begin{array}{ll}
S_{H H} & S_{H V} \\
S_{V H} & S_{V V}
\end{array}\right) \\
& \left(\begin{array}{cc}
\cos \Omega & \sin \Omega \\
-\sin \Omega & \cos \Omega
\end{array}\right)\left(\begin{array}{ll}
1 & \delta_{3} \\
\delta_{4} & f_{2}
\end{array}\right)+\left(\begin{array}{ll}
n_{H H} & n_{H V} \\
n_{V H} & n_{V V}
\end{array}\right)
\end{aligned}
$$

where $\mathbf{S}$ and $\mathbf{M}$ are the true and measured scattering matrices, $\mathbf{F}$ represents the one-way Faraday rotation matrix, $\mathbf{R}$ and $\mathbf{T}$ are the receive and transmit distortion matrices of the radar system. In $\mathbf{R}$ and $\mathbf{T}$, the diagonal terms ( $f_{1}$ and $f_{2}$ ) are channel imbalance and the off-diagonal terms $\left(\delta_{1}, \delta_{2}, \delta_{3}\right.$ and $\left.\delta_{4}\right)$ are cross-talk. A difference between airborne SAR and spaceborne SAR is an existence of Faraday rotation matrix in eq.(1). $\mathbf{n}$ is noise matrix.

A procedure for calibration of polarimetric SAR data that takes into account Faraday rotation is to estimate all parameters in $\mathbf{R}, \mathbf{T}$ and $\mathbf{F}$. These parameters are composed of six complex values and one real value. If $\mathbf{R}, \mathbf{T}$ and $\mathbf{F}$ are known and $\mathbf{n}$ is ignored, the true scattering matrix can be obtained as

$$
\mathbf{S}=\mathbf{F}^{-1} \mathbf{R}^{-1} \mathbf{M} \mathbf{T}^{-1} \mathbf{F}^{-1}
$$


In [4], a useful polarimetric calibration technique considering Faraday rotation was proposed by Dr. Freeman. In order to do polarimetric calibration, he uses a trihedral corner reflector and the natural distributed targets in a scene. The natural distributed targets are required to satisfy the reflection symmetry which means the co- and crosspolarized responses are uncorrelated as

$$
<S_{H H} S_{H V}^{*}>=<S_{V V} S_{H V}^{*}>=0
$$

where $<>$ denotes an ensemble average. Moreover, his technique assumes that the cross-talks of SAR system are negligible (-30dB). Thus $\mathbf{R}$ and $\mathbf{T}$ are simplified as follows

$$
\mathbf{R}=\left(\begin{array}{ll}
1 & 0 \\
0 & f_{1}
\end{array}\right), \quad \mathbf{T}=\left(\begin{array}{ll}
1 & 0 \\
0 & f_{2}
\end{array}\right) .
$$

In the next section, we explain a polarimetric calibration technique, which is based on a genetic algorithm (GA), to calibrate cross-talks, channel imbalances and Faraday rotation angle using one trihedral corner reflector and the measured polarimetric SAR data without considering reflection symmetry.

\section{POLARIMETRIC CALIBRATION METHOD USING GENETIC ALGORITHM}

Genetic algorithm can be used to find an approximated global optimal solution to a nonlinear optimization problem [5]. This algorithm prepares a set of candidate solutions (chromosomes) and evaluates the fitness of the objective function at each generation. If a fitness level is reached to a termination condition, it is considered that an approximated solution is found. In order to produce a next generation, genetic algorithm uses selection of mate, crossover and mutation as biological evolution. In the case polarimetric calibration, a chromosome consists of two channel imbalance, four cross-talks and Faraday rotation angle.

The true polarimetric SAR data is expressed as eq. (2) and consists of four polarimetric coefficients with respect to $\mathrm{HH}, \mathrm{HV}, \mathrm{VH}$ and VV. The covariance matrix $\mathbf{C}$ can be obtained from these polarimetric coefficients.

$$
\begin{aligned}
\mathbf{k} & =\left[\begin{array}{llll}
S_{H H} & S_{H V} & S_{V H} S_{V V}
\end{array}\right]^{T} \\
\mathbf{C} & =\left\langle\mathbf{k} \cdot \mathbf{k}^{\dagger}\right\rangle \\
& =\left[\begin{array}{llll}
\left\langle S_{H H} S_{H H}^{*}\right\rangle & \left\langle S_{H H} S_{H V}^{*}\right\rangle & \left\langle S_{H H} S_{V H}^{*}\right\rangle & \left\langle S_{H H} S_{V V}^{*}\right\rangle \\
\left\langle S_{H V} S_{H H}^{*}\right\rangle & \left\langle S_{H V} S_{H V}^{*}\right\rangle & \left\langle S_{H V} S_{V H}^{*}\right\rangle & \left\langle S_{H V} S_{V V}^{*}\right\rangle \\
\left\langle S_{V H} S_{H H}^{*}\right\rangle & \left\langle S_{V H} S_{H V}^{*}\right\rangle & \left\langle S_{V H} S_{V H}^{*}\right\rangle & \left\langle S_{V H} S_{V V}^{*}\right\rangle \\
\left\langle S_{V V} S_{H H}^{*}\right\rangle & \left\langle S_{V V} S_{H V}^{*}\right\rangle & \left\langle S_{V V} S_{V H}^{*}\right\rangle & \left\langle S_{V V} S_{V V}^{*}\right\rangle
\end{array}\right]
\end{aligned}
$$

where $\mathbf{k}$ is a target vector and $\dagger$ is a complex conjugate transpose. When a monostatic radar system is used to observe the terrains on earth, the scattering matrix needs to satisfy scattering reciprocity meaning that $S_{H V}$ is equal to $S_{V H}$. Thus, we can get some equations from (6).

$$
\begin{aligned}
& <S_{H V} S_{H V}^{*}>=<S_{V H} S^{*}{ }_{V H}>, \\
& <S_{H V} S_{V H}{ }^{*}=<S_{V H} S_{H V}{ }^{*}> \\
& \operatorname{Im}\left(<S_{H V} S_{V H}^{*}\right)=0
\end{aligned}
$$

Equations (7a)-(7c) have to be satisfied anywhere in polarimetric SAR data. We apply these equations to the objective function of genetic algorithm to find the polarimetric calibration parameters. Therefore, we do not need to use the reflection symmetry condition and the assumption that cross-talks are negligible. However, more equations are needed to obtain six complex and one real unknowns. In order to overcome this problem, we use a trihedral corner reflector information. A scattering matrix of the trihedral corner reflector is written as

$$
\mathbf{S}_{T r i}=\left(\begin{array}{ll}
1 & 0 \\
0 & 1
\end{array}\right)
$$

From equation (8), more equations are obtained.

$$
\begin{aligned}
& <S_{H H} S_{H H}^{*}>=<S_{V V} S^{*}{ }_{V V}>, \\
& <S_{H H} S^{*}{ }_{V V}>=<S_{V V} S_{H H}^{*} \\
& \operatorname{Im}\left(<S_{H H} S^{*}{ }_{V V}>\right)=0
\end{aligned}
$$

The objective function to evaluate the fitness based on reciprocity and trihedral corner reflector is defined based on eq.(7) and (9). A chromosome $\mathbf{D}_{\mathrm{m}}$ is expressed as

$$
\mathbf{D}_{m}=\left[\begin{array}{lllllll}
f_{1, m} & f_{2, m} & \delta_{1, m} & \delta_{2, m} & \delta_{3, m} & \delta_{4, m} & \Omega_{m}
\end{array}\right]
$$

where $\mathrm{m}$ is number of generation. A scattering matrix calibrated by $\mathbf{D}_{\mathrm{m}}$ is represented as

$$
\begin{aligned}
\mathbf{S}\left(\mathbf{D}_{\mathrm{m}}\right) & =\mathbf{F}\left(\mathbf{D}_{\mathrm{m}}\right)^{-1} \mathbf{R}\left(\mathbf{D}_{\mathrm{m}}\right)^{-1} \mathbf{M T}\left(\mathbf{D}_{\mathrm{m}}\right)^{-1} \mathbf{F}\left(\mathbf{D}_{\mathrm{m}}\right)^{-1} \\
& =\left(\begin{array}{cc}
S_{H H}\left(\mathbf{D}_{m}\right) & S_{H V}\left(\mathbf{D}_{m}\right) \\
S_{V H}\left(\mathbf{D}_{m}\right) & S_{V V}\left(\mathbf{D}_{m}\right)
\end{array}\right)
\end{aligned}
$$

An objective function $F\left(\mathbf{D}_{\mathrm{m}}\right)$ is derived based on eq.(7) and (9). If an area where trihedral corner reflector does not exist is selected to estimate $F\left(\mathbf{D}_{\mathrm{m}}\right)$, the objective function $F_{\text {no_cr }}\left(\mathbf{D}_{\mathrm{m}}\right)$ can be expressed as

$$
\begin{aligned}
F_{n o_{-} c r}\left(\mathbf{D}_{m}\right) & =\left|\frac{\left\langle S_{H V}\left(\mathbf{D}_{m}\right) S_{H V}^{*}\left(\mathbf{D}_{m}\right)\right\rangle-\left\langle S_{V H}\left(\mathbf{D}_{m}\right) S_{V H}^{*}\left(\mathbf{D}_{m}\right)\right\rangle}{\left\langle M_{H V} M_{H V}^{*}\right\rangle-\left\langle M_{V H} M_{V H}^{*}\right\rangle}\right| \\
& +\left|\frac{\left\langle S_{H V}\left(\mathbf{D}_{m}\right) S_{V H}^{*}\left(\mathbf{D}_{m}\right)\right\rangle-\left\langle S_{V H}\left(\mathbf{D}_{m}\right) S_{H V}^{*}\left(\mathbf{D}_{m}\right)\right\rangle}{\left\langle M_{H V} M_{V H}^{*}\right\rangle-\left\langle M_{V H} M_{H V}^{*}\right\rangle}\right| \\
& +\left|\frac{\operatorname{Im}\left(\left\langle S_{H V}\left(\mathbf{D}_{m}\right) S_{V H}^{*}\left(\mathbf{D}_{m}\right)\right\rangle\right)}{\operatorname{Im}\left(\left\langle M_{H V} M_{V H}^{*}\right\rangle\right)}\right|
\end{aligned}
$$


On the other hand, if an area where CR exists is selected, objective function $F_{\mathrm{cr}}\left(\mathbf{D}_{\mathrm{m}}\right)$ can be written as

$$
\begin{aligned}
F_{c r}\left(\mathbf{D}_{m}\right) & =\left|\frac{\left\langle S_{H H}\left(\mathbf{D}_{m}\right) S_{H H}^{*}\left(\mathbf{D}_{m}\right)\right\rangle-\left\langle S_{V V}\left(\mathbf{D}_{m}\right) S_{V V}^{*}\left(\mathbf{D}_{m}\right)\right\rangle}{\left\langle M_{H H} M_{H H}^{*}\right\rangle-\left\langle M_{V V} M_{V V}^{*}\right\rangle}\right| \\
& +\left|\frac{\left\langle S_{H H}\left(\mathbf{D}_{m}\right) S_{V V}^{*}\left(\mathbf{D}_{m}\right)\right\rangle-\left\langle S_{V V}\left(\mathbf{D}_{m}\right) S_{H H}^{*}\left(\mathbf{D}_{m}\right)\right\rangle}{\left\langle M_{H H} M_{V V}^{*}\right\rangle-\left\langle M_{V V} M_{H H}^{*}\right\rangle}\right| \\
& +\left|\frac{\operatorname{Im}\left(\left\langle S_{H H}\left(\mathbf{D}_{m}\right) S_{V V}^{*}\left(\mathbf{D}_{m}\right)\right\rangle\right)}{\operatorname{Im}\left(\left\langle M_{H H} M_{V V}^{*}\right\rangle\right)}\right|
\end{aligned}
$$

In order to reduce local minima, we use several estimation areas to calculate objective functions of eq.(12) and (13). Then final objective function becomes as follows.

$$
F\left(\mathbf{D}_{m}\right)=\sum_{k=1}^{K} F_{n o_{-} c r}\left(\mathbf{D}_{m}\right)+\sum_{l=1}^{L} F_{c r}\left(\mathbf{D}_{m}\right)
$$

where $K$ and $L$ are numbers of non CR areas and CR areas.

A flow chart of GA is shown in Fig.1. In case of complex variables, a crossover operation is done as

$$
\begin{aligned}
& I_{1}^{\prime}=c I_{1}+j(1-c) I_{2} \\
& I_{2}^{\prime}=c I_{2}+j(1-c) I_{1}
\end{aligned}
$$

where $c$ is a uniformly distributed random value over the range $[0,1]$ and $j=\sqrt{-1}$. A mutation operation is calculated based on ref.[5]. Moreover, in order to restrict a range of initial chromosome and to reduce local minima, the calculation scheme of GA becomes as follows.

Step 1) Calculate $f_{1}, f_{2}$ and $\Omega$ roughly from GA. At this time, cross-talks $\delta_{1}-\delta_{4}$ are assumed to be zero and the ranges of initial chromosome with respect to $f_{1}, f_{2}$ and $\Omega$ is set widely.

Step 2) Make new initial chromosome set with respect to $f_{1}, f_{2}$ and $\Omega$ in the restricted ranges based on results of Step 1) and make the parameters with respect to $\delta_{1}-\delta_{4}$ of initial chromosome set based on priori information that crosstalks are smaller than 1 .

Step 3) Calculate $f_{1}, f_{2}, \delta_{1}-\delta_{4}$ and $\Omega$ from GA using the initial chromosome set of Step 2).

The GA calculation is terminated when one chromosome gets a best fitness or after a certain fixed number generations is reached.

\section{EXPERIMETAL RESULTS}

To show the effectiveness of the proposed calibration method, Advanced Land Observing Satellite (ALOS) / Phased Array type L-band SAR (PALSAR) data was used. ALOS was launched on January 24, 2006, and PALSAR is one of three sensors loaded on ALOS. PALSAR is the first spaceborne L-band polarimetric SAR. PALSAR observed Nagasaki, Japan on December 23, 2007. The observation path was ascending. When PALSAR observed Nagasaki, one trihedral corner reflector was deployed in a playground of Nagasaki University. An echo of corner reflector is shown in Fig.2. The areas estimated in eq (12) consist of 6 areas $(25 \times 25$ pixel $)$ which are urban, mountain and firm areas. It means that $K$ is 6 and $L$ is 1 in eq.(14) To provide initial chromosomes, each parameter is chosen as $0.5 \leq|f| \leq 1.5$, $-180^{\circ} \leq|\operatorname{Arg}(f)| \leq 180^{\circ}$, and $-5^{\circ} \leq \Omega \leq 5^{\circ}$. The number of chromosome is 300 . The crossover probability is 0.5 and the mutation probability is 0.15 . The maximum number of generation is 500. However, the proposed GA algorithm has the problem that there is a possibility to provide wrong polarimetric calibration parameter sets with respect to $f_{1}$ and $f_{2}$. For example, correct and wrong parameter sets are as follows.

$$
\begin{aligned}
& \text { Correct case })\left\{\begin{array}{l}
f_{1}=0.71 \angle-0.6^{\circ} \\
f_{2}=0.98 \angle 21.0^{\circ}
\end{array}\right. \\
& \text { Wrong Case })\left\{\begin{array}{c}
f_{1}=0.71 \angle 179.4^{\circ} \\
f_{2}=0.98 \angle-159.0
\end{array}\right.
\end{aligned}
$$

Thus, in a calibrated scattering matrix, a $180^{\circ}$ sign ambiguity of $S_{\mathrm{HV}}$ and $S_{\mathrm{VH}}$ happens. In order to overcome this problem urban structure can be used. In built-up areas, radar polarization orientation shifts occur [7].

$$
\begin{aligned}
\text { Wall orientation angle }-90^{\circ}<\phi<0^{\circ}: \operatorname{Arg}\left(S_{\mathrm{HV}} S_{\mathrm{HH}}^{*}\right) \approx 0^{\circ} \\
0<\phi<90^{\circ}: \operatorname{Arg}\left(S_{\mathrm{HV}} S_{\mathrm{HH}}^{*}\right) \approx 180^{\circ}
\end{aligned}
$$

where $\phi$ is wall orientation angle as shown in Fig.3. Then we can get more equations. We add additional function for estimating eq.(16a,b) to objective function of eq.(14).

$$
\begin{aligned}
F_{\text {built-up }}\left(\mathbf{D}_{m}\right) & =\left|\frac{\operatorname{Arg}\left(\left\langle S_{H V}\left(\mathbf{D}_{m}\right) S_{H H}^{*}\left(\mathbf{D}_{m}\right)\right\rangle\right)-\text { theoretical_angle }}{\operatorname{Arg}\left(\left\langle M_{H V} M_{H H}^{*}\right\rangle\right)-\text { theoretical_angle }}\right| \\
& +\left|\frac{\operatorname{Arg}\left(\left\langle S_{V H}\left(\mathbf{D}_{m}\right) S_{H H}^{*}\left(\mathbf{D}_{m}\right)\right\rangle\right)-\text { theoretical_angle }}{\operatorname{Arg}\left(\left\langle M_{V H} M_{H H}^{*}\right\rangle\right)-\text { theoretical_angle }}\right|, \\
F\left(\mathbf{D}_{m}\right)= & \sum_{k=1}^{K} F_{n c_{-} r}\left(\mathbf{D}_{m}\right)+\sum_{l=1}^{L} F_{c r}\left(\mathbf{D}_{m}\right)+F_{\text {built }-u p}\left(\mathbf{D}_{m}\right)
\end{aligned}
$$

where theoretical angle $\left(0^{\circ}\right.$ or $\left.180^{\circ}\right)$ is determined on condition that $\phi$ is known in estimated built-up area. The estimation results are shown in Table 1 with JAXA(Japan Aerospace Exploration Agency)'s polarimetric calibration parameters. The parameter ranges of initial chromosome at step 2) are chosen as $0.61 \leq\left|f_{1}\right| \leq 0.81,-10.60^{\circ} \leq\left|\operatorname{Arg}\left(f_{1}\right)\right| \leq 9.4^{\circ}$, $0.88 \leq\left|f_{2}\right| \leq 1.08, \quad-11.0^{\circ} \leq\left|\operatorname{Arg}\left(f_{2}\right)\right| \leq 31.0^{\circ}, \quad 0.0 \leq|\delta| \leq 0.1, \quad-$ $180^{\circ} \leq|\operatorname{Arg}(\delta)| \leq 180^{\circ}$, and $-1.1^{\circ} \leq \Omega \leq 0.9^{\circ}$ based on the result of step 1). $f_{1}$, and $f_{2}$ are close to JAXA values. The crosstalks $\delta_{1-4}$ are estimated to be small. This feature is similar to that of JAXA cross-talks. Moreover, $\Omega$ is estimate to be $0.04^{\circ}$ by proposed GA method. We estimated $\Omega$ by Freeman 
method. His method shows that $\Omega$ is $-0.16^{\circ}$. Both methods provide similar Faraday rotation angle. Therefore, it is confirmed that the polarimetric calibration method using genetic algorithm can estimate not only channel imbalance and cross-talk of SAR system but also Faraday rotation angle simultaneously.

\section{CONCLUSIONS}

This paper presented a polarimetric calibration method using a genetic algorithm. This method can estimate all polarimetric calibration parameters including Faraday rotation angle and does not need a polarimetric data satisfying reflection symmetry property. We applied the method to the polarimetric L-band data acquired by PALSAR. Thus, channel imbalance and Faraday rotation angles of Nagasaki could be estimated. Moreover, crosstalks were evaluated to be small. This feature is similar to that of JAXA cross-talks.

\section{ACKNOWLEDGEMENT}

The author expresses their sincere appreciations to JAXA for providing PALSAR image data. The author is grateful that a trihedral corner reflector was borrowed from ERSDAC, Japan. This research was partially supported by the Ministry of Education, Science, Sports and Culture, Grant-in-Aid for Young Scientists (B) 20760267, 2008.

\section{REFERENCES}

[1] A. Freeman, "SAR calibration: An overview", IEEE Trans. Geosci. Remote Sens., vol. 30, No. 11, pp. 1107-1121, 1992.

[2] S. Quegan, "A unified algorithm for phase and cross-talk calibration of polarimetric data -Theory and observations", IEEE Trans. Geosci. Remote Sens., vol. 32, No. 1, pp. 89-99, 1994.

[3] M. Fujita, T. Masuda, Y. Fujino, M. Satake, "Polarimetric calibration of the SIR-C C-band channel using active radar calibrators and polarization selective dihedrals", IEEE Trans. Geosci. Remote Sens., vol. 32, No. 6, pp. 1872-1878, 1998.

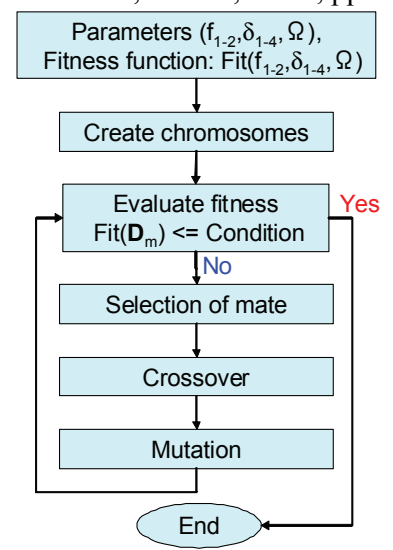

Fig. 1 A flow chart of GA algorithm.
[4] A. Freeman, "Calibration of Linearly Polarized Polarimetric SAR data Subject to Faraday Rotation", IEEE Trans. Geosci. Remote Sens., vol. 42, No. 8, pp. 1617-1624, 2004.

[5] W. Xiong, "Polarimetric Calibration Using a Genetic Algorithm", IEEE Geosci. Remote Sens. Letters, vol. 4, No. 3, pp. 421-425, 2007.

[6] T. L. Ainsworth, L.F.-Famil, J.-S. Lee, "Orientation Angle Preserving A Posteriori Polarimetric SAR Calibration", IEEE Trans. Geosci. Remote Sens., vol. 44, No. 4, pp. 994-1003, 2006.

[7] H. Kimura, "Calibration of spaceborne polarimetric SAR data using polarization orientation", Proc. of IGARSS 2006, Vol. 1, pp. 344-347, 2006.

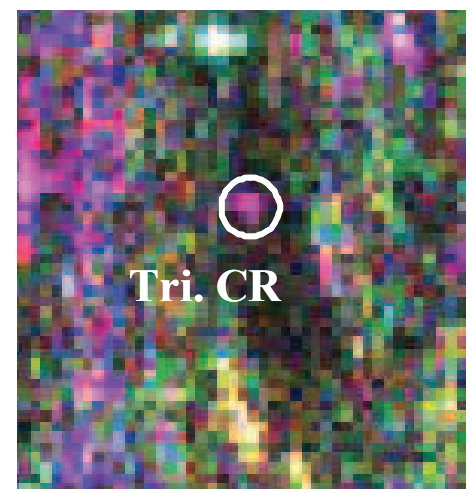

Fig. 2 Trihedral corner reflector echo deployed in playground of Nagasaki University.

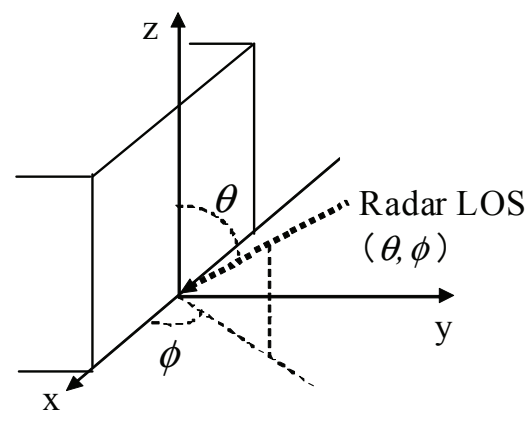

Fig. 3 Orientation angle of building wall.

Table 1 Polarimetric calibration parameters estimated by proposed method with JAXA's parameters.

\begin{tabular}{|c|c|c|c|}
\hline \multirow{2}{*}{} & \multicolumn{2}{|c|}{$\begin{array}{c}\text { Proposed method } \\
\text { (GA, Nagasaki) }\end{array}$} & $\begin{array}{c}\text { JAXA } \\
\text { (Quegan method, } \\
\text { Amazon) }\end{array}$ \\
\cline { 2 - 3 } & Step 1 & Step 2 & $0.72 \angle 1.88^{\circ}$ \\
\hline$f_{1}$ & $0.71 \angle-0.61^{\circ}$ & $0.69 \angle 0.03^{\circ}$ & $1.03 \angle 21.81^{\circ}$ \\
\hline$f_{2}$ & $0.98 \angle 21.0^{\circ}$ & $1.02 \angle 20.59^{\circ}$ & $0.010 \angle 131.48^{\circ}$ \\
\hline$\delta_{1}$ & & $0.007 \angle-140.17^{\circ}$ & $0.010 \angle 128.11^{\circ}$ \\
\hline$\delta_{2}$ & & $0.006 \angle 5.58^{\circ}$ & $0.013 \angle 79.37^{\circ}$ \\
\hline$\delta_{3}$ & & $0.004 \angle 113.50^{\circ}$ & $0.013 \angle-151.50^{\circ}$ \\
\hline$\delta_{4}$ & & $0.013 \angle-101.71^{\circ}$ & 0.013 \\
\hline$\Omega$ & $-0.11^{\circ}$ & $-0.04^{\circ}$ & \\
\hline
\end{tabular}

